\title{
The effect of moment arm length on high angled femoral neck fractures (Pauwels' III)
}

\author{
Matthew S. LePine ${ }^{1}$, William R. Barfield ${ }^{1,3}$, John D. DesJardins ${ }^{2}$, Langdon A. Hartsock ${ }^{1}$ \\ ${ }^{1}$ Department of Orthopaedic Surgery, Medical University of South Carolina, Charleston, SC; \\ ${ }^{2}$ Department of Bioengineering, Clemson University, Clemson, SC; \\ ${ }^{3}$ Health \& Human Performance, College of Charleston, Charleston, SC. \\ Email: lepine@musc.edu; jdesjar@,clemson.edu; hartsock@musc.edu; barfielb@musc.edu
}

Received 21 January 2010; revised 6 February 2010; accepted 8 February 2010.

\begin{abstract}
This study investigated loads among five fixation types (FT) [three cannulated screws (CS), dynamic hip screws with and without derotational screws (DHS-DS and DHS), and dynamic helical hip screws with and without derotational screws (DHHS-DS and DHHS)] across three fracture moment lengths (ML) in Pauwels' Type III fractures. Methods: Seventy-five sawbones were tested $(5 \mathrm{FT} \times 5$ trials $\times 3 \mathrm{ML})$. The study hypothesis was that significant differences in axial loading to failure would be demonstrated when CS was compared with the other four FT at the three MLs. Each construct was exposed to an axial compressive load to failure. Construct failure was defined as $5 \mathrm{~mm}$ of migration at the fracture site or fixation failure. Shapiro-Wilk was used to test for data normality. Subsequently, independent t-tests with Bonferroni correction was used for paired comparisons. Results: At fracture Moments A and B there were no statistical differences between CS and the other FT. At fracture Moment $C$ all four FT yielded significantly higher $(p \leq 0.001)$ loads compared with $C S$. Conclusions: for basicervical fractures CS is a suboptimal form of fixation compared with DHS and DHHS both with and without derotation screws.
\end{abstract}

Keywords: Pauwels' Fracture, Biomechanical Testing, Fixation

\section{INTRODUCTION}

Pauwels' Type III fracture is described as a fracture angle of the femoral neck and shaft greater than $50^{\circ}$ in the coronal plane, [1-5] with dominating shear stress and varus loading and less compressive loading and stress $[2,3]$. As a consequence, internal fixation of these unstable fractures surgically has been associated with high rates of non unions $[3,6]$ sparking debate over the most effective internal fixation technique [7-10]. Biomechanical and clinical studies have shown noncomminuted femoral neck fractures in the subcapital and transcervical regions fixed with three cannulated screws yield optimal stabilization results, [11-13] while fractures in the basicervical region show best results when fixed with a sliding hip screw $[13,14]$.

Other variables contribute to the nonunion rates of fixed femoral neck fractures including; femoral neck fracture type, vascular insufficiency, and inaccurate reduction [15]. Stankewich et al. expanded this list to include femoral head bone density, percent comminution of the fracture surface, moment arm length, and orientation angle of the fracture surface relative to the femoral shaft [8].

Although biomechanical tests have compared the effectiveness of various fixation devices for different fractures, [16] no study has sought to make a direct comparison based on moment arm length while maintaining a constant fracture angle.

The purpose of this study was to compare the failure load of four different methods of fixation; Dynamic Hip Screws with (DHS-DS) and without derotational screws (DHS), and Dynamic Helical Hip Screw with (DHHSDS) and without derotational screws (DHHS) (Synthes USA, West Chester, PA) for Pauwels' Type III fracture across three different fracture moment lengths with three cannulated screws (CS), the traditional "gold standard" [17-19]. The study hypothesis was that no statistically significant differences in resistance to axial loading to failure would be demonstrated when the CS was compared with the other four fixation types at each of the three moment lengths.

\section{MATERIALS AND METHODS}

Seventy-five new $3^{\text {rd }}$ Generation Composite left femoral sawbones (Sawbones Worldwide-Pacific Research Laboratories, Inc. Vashon, WA) of equal density was utilized for this experiment. The $3^{\text {rd }}$ Generation Composite Bone model is designed to simulate natural cortical bone through use of glass fibers and epoxy resin pressure injected around a foam core. Density of the sawbones was 
$1.7 \mathrm{~g} / \mathrm{cc}$ with $90 \mathrm{MPa}$ of tensile strength and a modulus of $12.4 \mathrm{GPa}$. The compressive strength was $120 \mathrm{MPa}$ and the modulus was 7.6 GPa. Other investigators [20,21] have found that $3^{\text {rd }}$ Generation Composite femurs fall within the normal range for bending, torsional and axial loading compared with both fresh-frozen and dried rehydrated cadaver bone. Sawbones also show less variability compared with cadaveric specimens, with the interfemur variability of composites being 20-200 times lower than that for cadaveric specimens, thereby allowing for smaller differences to be characterized with small sample sizes $[20,21]$. Our apriori power analysis supported use of 5 samples/fixation/moment arm length. Five sawbones for each fracture-fixation and moment arm length were performed, resulting in a total of 75 constructs being tested ( 5 fixation types $\times 5$ sawbones/fixation type $\times 3$ moment arm lengths).

The three groups of sawbone fractures were created, based on the fracture moment arm length (Figure 1; $\mathrm{A}=2.0$ $\mathrm{cm}, \mathrm{B}=3.1 \mathrm{~cm}, \mathrm{C}=4.2 \mathrm{~cm}$ ). The moment arm distance was measured from the femoral head center to the center of the fracture line on the sawbone specimen using Vernier calipers as shown in Figure 1. A custom cutting guide was engineered to ensure fracture line reproducibility.

A femoral neck osteotomy at $70^{\circ}$ to the horizontal (Figure 1), was created by a single investigator with a bandsaw in each of the sawbones, extending from the superior femoral neck to subcapital, transcervical and basicervical regions. All fractures were repaired by a single investigator using one of five forms of fixation.

The three CSs ( 7.3 cannulated screw $100 \mathrm{~mm}, 16 \mathrm{~mm}$ thread-Synthes, Paoli, PA, USA Product \# 208.900) were placed in an inverted triangle configuration at a $135^{\circ}$. A cannulated screw set jig supplied by Synthes was used for screw insertion to reduce variability.

The four other fixation types included: 1) DHS-DS (4 hole $135^{\circ}$ Std plate-Synthes Product \# 281.140, $90 \mathrm{~mm}$ compression lag screw-Synthes Product \# 280.900) with a derotational screw (7.3 cannulated screw $100 \mathrm{~mm}, 16$ mm thread-Synthes Product \# 208.900); 2) DHS (4 hole $135^{\circ}$ Std plate-Synthes Product \# 281.140, 90mm screw-Synthes Product \# 280.900) without a derotational screw; 3) DHHS (4 hole $135^{\circ}$ Std LCP-PlateSynthes Product \# 282.612, $100 \mathrm{~mm}$ helical screw-Synthes Product \# 282.239) with a derotational screw (7.3 cannulated screw $100 \mathrm{~mm}, 16 \mathrm{~mm}$ thread-Synthes Product \# 208.900); 4) DHHS-DS (4 hole $135^{\circ}$ std LCP-Plate-Synthes Product \# 282.612, $100 \mathrm{~mm}$ helical Screw-Synthes Product \# 282.239) without a derotational screw. All hardware was inserted according to the technique guide provide by the manufacturer. The 4-hole plate has traditionally been used for fixation of proximal femur fractures. A PMMA appliance (Figure 2) was created which cupped the displaced femoral head and proximal femoral neck to insure reduction accuracy. Implants were properly positioned by direct visualization on the surface of the bone. The hip lag screw guide wire was consistently placed in the center of the femoral head through use of an aiming device.

The angle of implant insertion was in accordance with the technique guide provided by the manufacturer including pre-drilling of all holes prior to fixation (Figure 3). A representative group of each fixation type was $\mathrm{X}$ rayed to insure anatomic reduction.

The femurs were uniformly potted in dental stone (Modern Materials) at $25^{\circ}$ of adduction in the coronal plane and neutral position in the sagittal plane to simulate the terminal stance phase of gait [22-25]. The Instron Mechanical Testing System (Instron Bi-Axial Servo-Hydraulic Testing Machine Model \# 8874-Norwood, MA) incrementally loaded the femoral head with an evenly applied compressive axial load through a polished stainless steel cup attached to the ram. Potted femurs were securely locked in place with the appliance pictured in Figure 4 for testing. The appliance was free to move in the medio-lateral plane.

The loading protocol utilized was from Chang et al., 1987 [25], designed to simulate single leg stance. In this

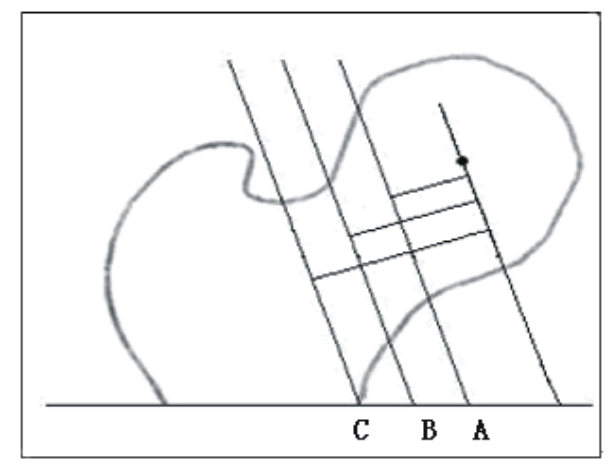

Figure 1. Schematic demonstration of moment arm length $(\mathrm{A}, \mathrm{B}, \mathrm{C}) . \mathrm{A}=$ Subcapital; $\mathrm{B}$ $=$ Transcervical $\mathrm{C}=$ Basicervical.

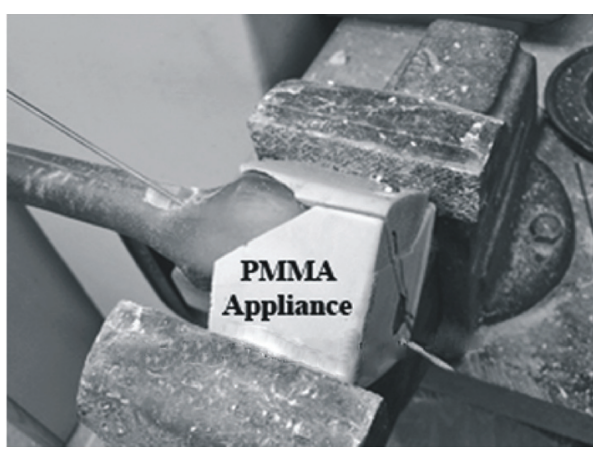

Figure 2. PMMA appliance used to hold the 2-part Pauwels' fracture in the vise for accurate reduction of fractures. 


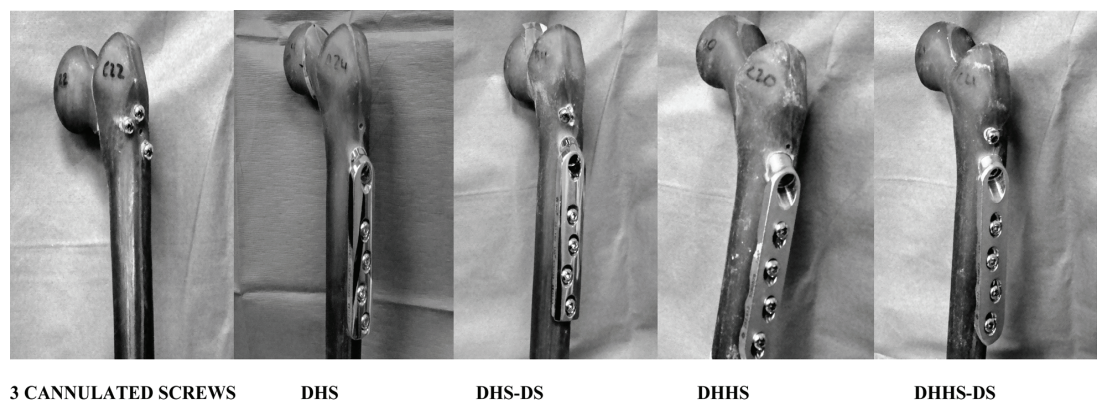

Figure 3. Pictures of the five fixation forms.

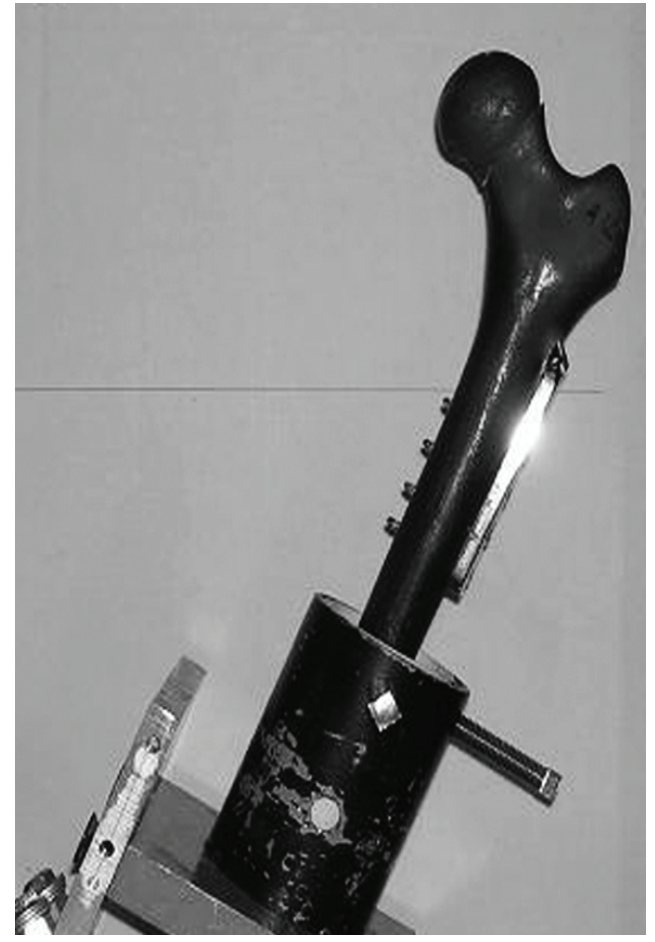

Figure 4. Testing jig used for mechanical testing of constructs.

single-leg phase, body weight is transferred ahead of the forefoot, creating loading conditions of nearly five times body weight [26]. Constructs were positioned under a servohydrolic materials testing system producing a vertically-downward force. Displacement was applied at a rate of $0.5 \mathrm{~mm} / \mathrm{s}$ and terminated when the construct reached failure criteria. Failure was defined as $5 \mathrm{~mm}$ of migration at the fracture site through either translation and/or a combination of migration and rotation of the femoral head or catastrophic failure of device or sawbone [13].

Force data were captured using Instron's Max v7.1 computer software at $100 \mathrm{~Hz}$. Statistical analysis was performed using SPSS Version 14 software. ShapiroWilk was utilized to test for normality. Unpaired t-tests were subsequently used to compare the four constructs;
DHS, DHS-DS, DHHS, and DHHS-DS to the CS constructs at each of the three moment lengths. Due to multiple unpaired comparisons a Bonferroni adjustment was used. Our apriori alpha level was $\mathrm{p} \leq 0.05$. With four comparisons the adjusted alpha was lowered to $\mathrm{p} \leq$ 0.013 .

\section{RESULTS}

Shapiro-Wilk test for normality indicated that the data was normally distributed $(>0.05)$. At fracture Moment A (subcapital) there was no statistical difference in yield forces $(p=0.026)$ (Figure 5(a)). At fracture Moment B (transcervical) there were no statistical differences in yield forces $(\mathrm{p}=0.022)$. (Figure 5(b)) At fracture Moment $\mathrm{C}$ (basicervical) all four fixation forms yielded statistically significant $(\mathrm{p} \leq 0.001)$ high axial loads when compared with CS as seen in Figure 5(c).

\section{DISCUSSION}

In the present investigation five fixation types at three different fracture moment lengths (A [subcapital], B [transcervical], C [basicervical]), were axially loaded and biomechanically testing in 75 sawbone models. Our study hypothesis was that no statistically significant differences in resistance to axial loading to failure would be demonstrated when the CS was compared with the other four fixation types at each of the three moment lengths. Results supported our hypotheses at each moment length except the basicervical model where statistically different results were seen

Aminian et al. in the $J$ Orthop Trauma in 2007, biomechanically tested 32 cadaver specimens utilizing four fixation types. Two of our fixation types were the same as Aminian et al. (CS and DHS). Aminian et al. specimens were cyclically loaded at $1400 \mathrm{~N}$ at $3 \mathrm{~Hz}$ for 10,000 cycles followed by a load-to failure test. All CS specimens failed during incremental loading with a failure load of $862 \mathrm{~N}$ [16]. Tan et al. tested five pairs of fresh frozen cadaver femurs and used cyclical axial loading of $750 \mathrm{~N}$ at $0.5 \mathrm{~Hz}$ for 200 cycles [27] and found that the load at the yield point was significantly higher in the group with more horizontally oriented screws leading to 


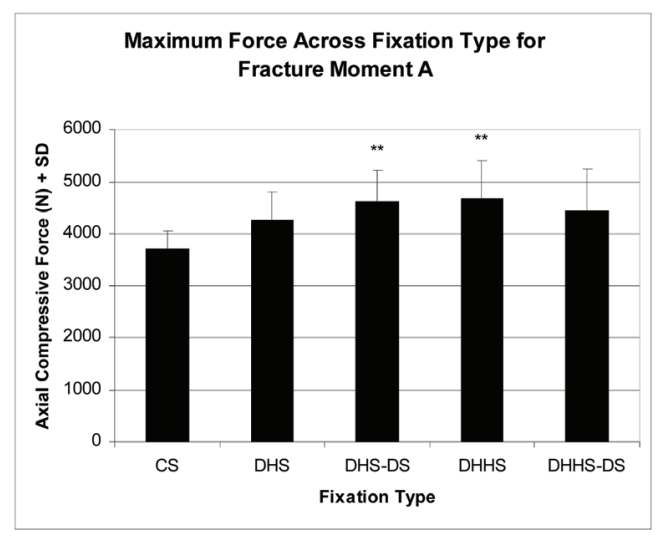

(a)

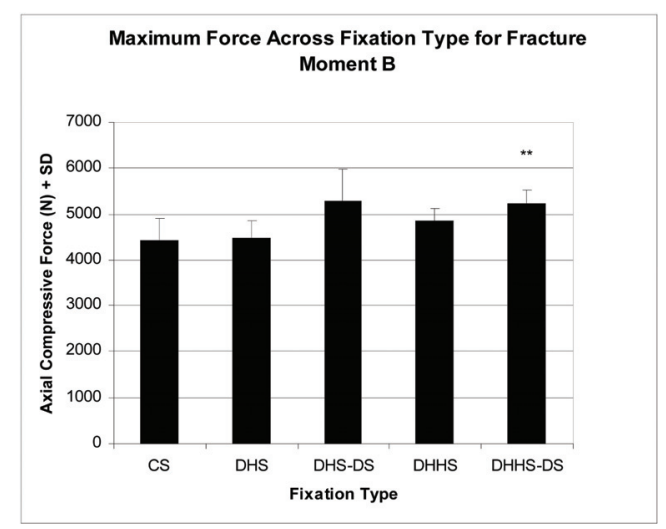

(b)

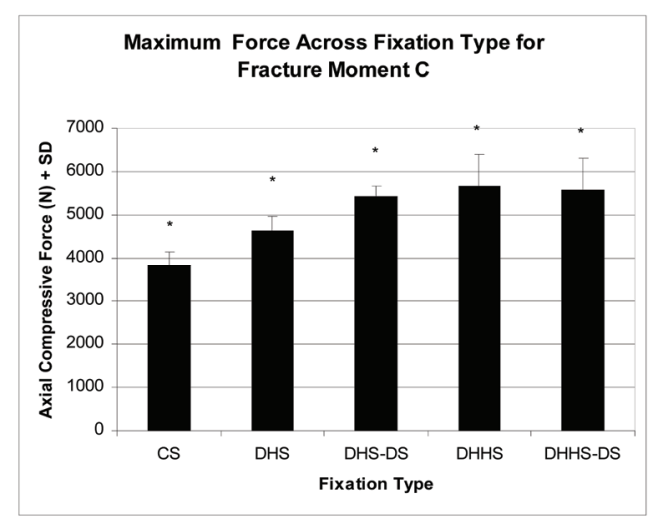

(c)

Figure 5. Maximum axial force for fracture Moment $\mathrm{A}^{* *}=$ statistical trend compared with CS.

the suggestion that two horizontal screws in the femoral neck provide better stiffness.

In our study both the CS and DHS construct failed at higher loads ([Moment A-3709.9 N] [Moment B-4423.4 $\mathrm{N}]$ [Moment C-3830.8 N]), which we partially attribute to the lack of cyclical fatigue testing, specimen differences (cadaver bone vs. sawbones of uniform density) and different mechanical testing based on our moment arm length differences and different fixation types. The moment arm distance, measured from the center of the femoral head to the fracture line, plays a key role. As the distance increases the torque across the fracture line increases. The authors of the present work evaluated the current English literature through traditional search techniques and could not locate any studies that have investigated the mechanical properties of various fixation techniques in Pauwels' Fracture Type III with moment length considered.

Our testing protocol from Chang et al., 2002 [25], best approximated the study goal of assessing maximal axial compressive loading leading to eventual failure among the fixation types, although other protocols exist [27-30]. The authors recognized through earlier unpublished pilot data that there would be minimal flexure of the sawbone, similar to what was observed in Aminian et al.

Our fracture moments results supported the null hypothesis are at the subcapital and transcervical region, but not at the basicervical region.

The fracture Moment $\mathrm{C}$ model yielded statistically significant differences $(p<0.001)$ between CS and each of the other four fixation types. Our results, based on the use of synthetic femurs, suggest that for a subcapital high angle fracture (Moment A) the DHHS and DHS-DS may be better clinical choices for fixation than the standard CS and at the intermediate fracture Moment B the DHHS-DS would be the preferred form of fixation although statistically significant differences did not exist. At fracture Moment $\mathrm{C}$, a high angle basicervical fracture, the study results suggest that all of the fixed angle fixation forms that we tested are superior to CS in combating axial load. At Moment C, there is a shorter amount of screw length supported by the intact femur and when a force is applied the screw is not supported by adjacent cortical bone due to the width at the base of the femoral neck. Fixation with CS can lead to greater displacement under the applied load.

The literature is inconclusive with respect to DHS fixation. Baitner et al. demonstrated that the DHS construct was significantly stronger than the cannulated screw construct [13], however Husby et al., found no significant differences between the same two constructs [10].

In a normal gait cycle at heel strike and toe off, the hip experiences approximately 4-5 times body weight [24]. In the instance of a $100 \mathrm{~kg}(981 \mathrm{~N})$ individual walking, the hip joint can experience between 3924$4905 \mathrm{~N}$. These numbers approximate the values at failure found in our investigation. At fracture Moments A, B, and $\mathrm{C}$ the loads for all fixation types are at or exceed the upper range of axial loading compared with the example provided for normal gait. Obviously, an in vitro study and what occurs in vitro is much different due to soft tissue damping which occurs in humans as we independently ambulate. However, our biomechanical results 
suggest that fixation type is a function of the moment arm length in Pauwels; Type III fracture.

The US population is getting older and progressively more obese. A $120 \mathrm{~kg}(1177 \mathrm{~N})$ patient can experience 4708-5885 $\mathrm{N}$ in the hip which surpasses the load bearing capacity of several of the fixation forms tested independent of moment arm length. In the event of a stumble, forces in the hip joint approach eight times body weight [24]. The result of this could be catastrophic based on the results demonstrated by this laboratory study.

The results found in this project are clinically relevant because we have demonstrated that significant differences do exist across moment lengths and fixation types in Pauwels Type III fractures. Even with adequate reductions, failure rates are higher with cannulated screws than with fixed angle devices [7]. Probe and Ward [31] reported that both fixed angle and multiple screws with divergent paths were superior to parallel screws. The current work supports the prior body of knowledge associated with the difficult clinical issue of surgical repair of Pauwels' Type III fractures.

Strengths of our study include: bone uniformity, fracture accuracy, a single investigators placement of screws and other types of fixation, and uniformity of testing and recording of data. The weaknesses of our investigation include uniaxial loading. One perceived weakness among some readers may be that we used sawbones as opposed to fresh cadaver femurs. While this perspective has merit, our position is that by using a material that possesses uniformity, our protocol measured the axial loading of the constructs and was not dependent on human bone quality in terms of density. Varying bone densities can introduce a confounding variable, which we chose to control in our work. This position is supported by studies reported in 1996 and 2001 using sawbones and comparing the mechanical results with human cadaver bone. The findings in these studies support the structural equivalent of composite bones with natural bones in axial, bending and torsional loading [20,21]. Our loading protocol at failure approximated load during single leg stance. Because of hip musculature stabilization our fixation model does not completely replicate in situ loading. Different directional force vectors can negatively affect fixation. Static loading in a uniaxial direction does not mimic the complex tensile, compressive and shear loads across the hip in situ.

This study is clinically relevant in that for subcapital high angle fractures differences existed with the DHHS and DHS-DS being better fixation choices when compared with the CS construct. For transcervical fractures the best choice was DHHS-DS and for high angle basicervical fractures DHS, DHS-DS, DHHS, and DHHSDS were all axially superior to CS for fracture fixation. However, as noted in a recent clinical paper that evaluated the efficacy of internal fixation of Pauwels' Type III femoral neck fractures, despite advances clinically and biomechanically the ideal fixation form remains unclear. Clearly patient bone quality, patient age and implant position all affect fixation results in patients as noted in a recent clinical paper [32].

Finally, our model was used purely as a model for comparison of stability of individual types of internal fixation. Our in vitro study results suggest that surgical fixation for Pauwels' Type III unstable fractures is a function of the moment arm distance and loading magnitude.

\section{REFERENCES}

[1] Nilsson, L., Johansson, A. and Stromqvist, B. (1993) Factors predicting healing complications in femoral neck fractures: 138 patients followed for 2 years. Acta Orthopaedica Scandinavica, 64(2), 175-177.

[2] Parker, M.J. and Dynan, Y. (1998) Is Pauwels' classification still valid? Injury, 29(7), 521-523.

[3] Bartonicek, J. (2001) Pauwels' classification of femoral neck fractures: Correct interpretation of the original. Journal of Orthopaedic Trauma, 15(5), 358-360.

[4] Pauwels, F. (1976) Biomechanics of the normal and diseased hip. Springer, Berlin.

[5] Caviglia, H.A., Osorio, P.Q. and Comando D. (2002) Classification and diagnosis of intracapsular fractures of the proximal femur. Clinical Orthopaedics and Related Research, 399(7), 17-27.

[6] Marti, R.K., Schuller, H.M. and Raaymakers, E.L. (1989) Intertrochanteric osteotomy for non-union of the femoral neck. Journal of Bone and Joint Surgery, 71(5), 782-787.

[7] Haidukewych, G.J., Liporace, F. and Gaines, R. (2005) Pauwels' type 3 vertical femoral neck fractures - What is the best fixation device? Orthopaedic Trauma Association 21 st Annual Meeting, 21(8), 2005, 130-131.

[8] Stankewich, C.J., Chapman, J., Muthusamy, R., Quaid, G., Schemitsch, E., Tencer, A. and Ching, R.P. (1996) Relationship of mechanical factors to the strength of proximal femur fractures fixed with cancellous screws. Journal of Orthopaedic Trauma, 10(4), 248-257.

[9] Weinrobe, M., Stankewich, C.J., Mueller, B., Tencer, A.F. (1998) Predicting the mechanical outcome of femoral neck fractures fixed with cancellous screw: An in vivo study. Journal of Orthopaedic Trauma, 12(1), 27-37.

[10] Husby, T., Alho, A., Hoiseth, A. and Fonstelien, E. (1987) Strength of femoral neck fixation, comparison of six techniques in cadavers. Acta Orthopaedica Scandinavica, 58(6), 634-637.

[11] Barnes, R., Brown, J.T., Garden, R.S. and Nicoll, E. (1976) Al subcapital fractures of the femur. Journal of Bone and Joint Surgery, 58B(1), 2-24.

[12] Bout, C.A., Cannegieter, D.M. and Juttman, J.W. (1997) Percutaneous cannulated screw fixation of femoral neck fractures: The three point principle. Injury, 28(2), 135-139.

[13] Baitner, A.C., Maurer, S.G., Hickey, D.G., Jazrawi, L.M., Kummer, F.J., Goldman, S. and Koval, K.J. (1999) Vertical shear fractures of the femoral neck. Clinical Orthopaedics \& Related Research, 367(65), 300-305.

[14] Deneka, D.A., Simonian, P., Stankewich, E.D., Chapman, J.R. and Tencer, A.F. (1997) Biomechanical comparison 
of internal fixation techniques for the treatment of unstable basicervical femoral neck fractures. Journal of Orthopaedic Trauma, 11(2), 337-343.

[15] LaVelle, D.G. (2003) Delayed union and nonunion of fractures. In: Canale, S.T., Ed., Campbell's Operative Orthopaedics, St. Louis, (10)3, 3125-3165.

[16] Aminian, A., Gao, F., Fedoriw, W.W., Zhang, L.Q., Kalainov, D.M. and Mark, B.R. (2007) Vertically oriented femoral neck fractures:mechanical analysis of four fixation techniques. Journal of Orthopaedic Trauma, 21(8), 544-548.

[17] Hernefaulk, L. and Messner, K. (1995) Femoral stiffness after osteosynthesis of a subcapital osteotomy in osteoporotic bone: An in vitro comparison of nine fixation methods. Journal of Bone and Joint Surgery, 9(58B), 224, 464-469.

[18] Kuokkanen, H., Korkala, O., Antti-Poika, I., Tolonen, J., Lehtimaki, M.Y. and Silvennoinen, T. (1991) Three cancellous bone screws versus a screw-angle plate in the treatment of Garden I \& II fractures of the femoral neck. Acta Orthopaedica Belgium, 57(1), 53-57.

[19] Parker, M.J. and Blundell, C. (1998) Choice of implant for internal fixation of femoral neck fractures: Metaanalysis of 25 randomized trials including 4,925 patients. Acta Orthopaedica Scandinavica, 69(2), 138-143.

[20] Christofolini, L., Vineconti, M., Cappello, A. and Toni, A. (1996) Mechanical validation of whole bone composite femur models. Journal of Biomechanics, 29(4), 525-535.

[21] Heiner, A.D. and Brown, T.D. (2001) Structural properties of a new design of composite replicate femurs and tibias. Journal of Biomechanics, 34(6), 773-781.

[22] Choueka, J., Koval, K.J., Kummer, F.J., Crawford, G. and Zuckerman, J.D. (1995) Biomechanical comparison of the sliding hip screw and the dome plunger: Effects of material and fixation design. Journal of Bone and Joint Surgery, 77-B(2), 277-283.

[23] Goh, J.C.H., Shah, K.M. and Bose, K. (1995) Biomechanical study on femoral neck fracture fixation in relation to bone mineral density. Clinical Biomechanics, 10(6), 304-308.
[24] Kubiak, E.N., Bong, M., Park, S.S., Kummer, F., Egol, K. and Koval, K.J. (2004) Intramedullary fixation of unstable intertrochanteric hip fractures: One or two lag screws. Journal of Orthopaedic Trauma, 18(1), 12-17.

[25] Chang, W.S., Zuckerman, J.D., Kummer, F.J. and Frankel, V.H. (1987) Biomechanical evaluation of anatomic reduction versus medial displacement osteotomy in unstable intertrochanteric fractures. Clinical Orthopaedics \& Related Research, 225(6), 141-146.

[26] (1986) Gait analysis. In: McAinsh, T.F., Ed., Physics in Medicine \& Biology Encyclopedia: Medical Physics, Bioengineering and Biophysics, Pergamon Press Inc, New York, 1(5), 356.

[27] Tan, V., Wong, K.L., Born, C.T, Harten, R. and DeLong, W.G. (2007) Two-screw femoral neck fracture fixation: A biomechanical analysis of 2 different confirurations. American Journal of Orthopedics, 36(9), 481-485.

[28] Chaim, S.H., Mukherjee, D.P., Ogden, A.L., Mayeux, R. H., Sadasivan, K.K. and Albright, J.A. (2002) A biomechanical study of femoral neck fracture fixation with the VHS-Vari-Angle hip fixation system. American Journal of Orthopedics, 31(Suppl 1), 22-24.

[29] Aboulafia, A.J., Price, M.M., Kennon, R.E. and Hutton, W.C. (1999) A comparison of mechanical strength of the femoral neck following locked intramedullary nailing using oblique versus transverse proximal screws. Journal of Orthopaedic Trauma, 13(3), 160-163.

[30] Kukla, C., Gaebler, C., Pichl, R.W., Prokesch, R., Heinze, G. and Heinz, T. (2002) Predictive geometric factors in a standardized model of femoral neck fractures. Experimental study of cadaveric human femurs. Injury, 33, 427-433.

[31] Probe, R. and Ward, R. (2006) Internal fixation of femoral neck fractures. Journal of the Academy of Orthopaedic Surgeons, 14(9), 565-571.

[32] Liporace, F., Gaines, R., Collinge, C. and Haidukewych, G.J. (2008) Results of internal fixation of Pauwels' type-3 vertical femoral neck fractures. Journal of Bone and Joint Surgery, 90(A), 1654-1659. 